\title{
Avaliação de serious game em programa de enfrentamento da obesidade infantil
}

Serious game assessment in a child obesity tackling program

Evaluación de serious game en programa de combate a la obesidad infantil

Jéssica David Dias ${ }^{1}$ ib https://orcid.org/0000-0002-4294-3686

Chris Mayara Tibes-Cherman ${ }^{1}$ id https://orcid.org/0000-0002-6653-4609

Rafaela Belém Aragão ${ }^{2}$ iD https://orcid.org/0000-0002-0889-6126

Hugo Tourinho Filho ${ }^{2}$ E hitps://orcid.org/0000-0001-6517-064X

Silvia Helena Zem-Mascarenhas ${ }^{3}$ ic https://orcid.orgy/0000-0001-7753-6725

Luciana Mara Monti Fonseca ${ }^{1}$ ib https://orcid.org/0000-0002-5831-8789

Como citar:

Dias JD, Tibes-Cherman CM, Aragão RB, Tourinho Filho $\mathrm{H}$, Zem-Mascarenhas SH, Fonseca LM.

Avaliação de serious game em programa de enfrentamento da obesidade infantil. Acta Paul Enferm. 2021;34:AAPE001045.

DOI

http://dx.doi.org/10.37689/actaape/2021A0001045

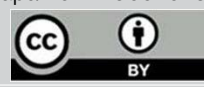

Descritores

Criança; Saúde da criança; Obesidade pediátrica; Sobrepeso; Comportamento alimentar; Jogos de vídeo; Adaptação psicológica

Keywords

Child; Child health; Pediatric obesity; Overweight; Feeding behavior; Video games; Adaptation,

psicological

Descriptores

Niño; Salud del niño; Obesidad pediátrica; Sobrepeso; Conducta alimentaria; Juegos de vídeo; Adaptación psicológica

Submetido 5 de Maio de 2020 Aceito 1 de Março de 2021

Autor correspondente Jéssica David Dias Email: jessdias@usp.br

\section{Resumo}

Objetivo: Avaliar um serious game enquanto estratégia educativa para crianças em um programa de enfrentamento de sobrepeso e obesidade.

Métodos: Pesquisa metodológica, com abordagem quantitativa e de corte transversal. Participaram do estudo as crianças pertencentes a um programa de condicionamento físico com games interativos para crianças obesas e/ou sobrepeso $(n=13)$. Foi feito o perfil dos participantes por meio de dados antropométricos, questionários sobre seus hábitos alimentares e de atividade física. Posteriormente, o serious game DigesTower foi apresentado como uma estratégia adicional ao programa e realizada a avaliação do jogo pelo público-alvo.

Resultados: Participaram crianças de sete a 11 anos com sobrepeso e obesidade. 0 DigesTower foi bem recebido pelos participantes, que afirmaram ter imersão, motivação e estímulo para melhoria do seu conhecimento. A versão final do jogo foi desenvolvida e disponibilizada online de forma gratuita e pública.

Conclusão: 0 estudo buscou incentivar 0 uso de ferramentas inovadoras para auxiliar como intervenções educativas no âmbito da obesidade infantil e o serious game foi avaliado como uma potencial estratégia educativa para crianças. Almeja-se que este estudo inspire futuras pesquisas em que seus objetos de estudo sejam 0 desenvolvimento de jogos digitais para o público infantil.

\section{Abstract}

Objective: To assess a serious game as an educational strategy for children in a program to tackle overweight and obesity.

Methods: This is methodological, quantitative and cross-sectional research. Children from a physical conditioning program with interactive games for obese and/or overweight children participated in the study $(n=13)$. Participants' profile was made through anthropometric data, questionnaires about their eating habits and physical activity. Subsequently, the serious game DigesTower was presented as an additional strategy to the program and the game was assessed by the target audience.

Results: Children aged seven to 11 years with overweight and obesity participated. DigesTower was well received by the participants, who claimed to have immersion, motivation and encouragement to improve their knowledge. The final version of the game was developed and made available online for free and publicly

Conclusion: The study sought to encourage the use of innovative tools to assist as educational interventions in the field of childhood obesity and the serious game was assessed as a potential educational strategy for children. It is hoped that this study will inspire future research in which its objects of study are the development of digital games for children. 


\section{Resumen}

Objetivo: Evaluar un serious game como estrategia educativa para niños en un programa de combate al sobrepeso y a la obesidad.

Métodos: Investigación metodológica, con enfoque cuantitativo y de corte transversal. Participaron en el estudio niños que forman parte de un programa de acondicionamiento físico con games interactivos para niños obesos o con sobrepeso $(n=13)$. El perfil de los participantes fue elaborado mediante datos antropométricos, cuestionarios sobre sus hábitos alimentarios y de actividad física. Luego se presentó el serious game DigesTower como una estrategia adicional del programa, y el público destinatario realizó la evaluación del juego.

Resultados: Participaron niños de 7 a 11 años con sobrepeso y obesidad. El DigesTowerfue bien recibido por los participantes, que afirmaron tener inmersión, motivación y estímulo para mejorar sus conocimientos. La versión final del juego fue desarrollada y se puso a disposición en línea de forma gratuita y pública.

Conclusión: El estudio buscó incentivar el uso de herramientas innovadoras para ayudar con intervenciones educativas en el ámbito de la obesidad infantil, y el serious game fue evaluado como una posible estrategia educativa para niños. Se espera que este estudio inspire futuras investigaciones que tengan como objeto de estudio el desarrollo de juegos digitales para el público infantil.

\section{Introdução}

Atualmente a obesidade infantil alcança proporçôes preocupantes em diversos países e retrata um problema a ser enfrentado. Esta é uma condição complexa, relacionada a fatores genéticos, nutricionais, socioeconômicos e ambientais e pode ser definida como o acúmulo de tecido gorduroso tanto de modo localizado quanto generalizado. ${ }^{(1)}$

Nas últimas décadas, as taxas de obesidade infantil aumentaram em todo o mundo e continuam a crescer especialmente em países de baixa ou média renda. $\mathrm{O}$ número de crianças e adolescentes com sobrepeso e obesos cresceu mais de dez vezes, de 11 milhóes em 1975 para 124 milhóes em 2016. (2) Mundialmente, prevê-se que, até 2025, aproximadamente 268 milhóes de crianças e adolescentes entre cinco a 17 anos teráo excesso de peso e deste total estima-se que 91 milhôes serão obesos. ${ }^{(3)}$

Estudos realizados na América do Sul mostraram a prevalência de sobrepeso de aproximadamente $7 \%$ em crianças menores de cinco anos, já em crianças com idade escolar a prevalência de sobrepeso e obesidade variou de $15 \%$ a $36,9 \%$, e entre adolescentes variou de $16,6 \%$ a $38 \%{ }^{(4,5)}$

As altas taxas de prevalência de sobrepeso e obesidade juntamente com os seus riscos tornam a doença um problema de saúde pública. Para combater a obesidade, organizaçóes de saúde do Brasil e do mundo têm estabelecido metas com vistas à promoção da saúde e incentivo a hábitos mais saudáveis. Os países das Américas deram um importante passo na luta contra a crescente epidemia de obesidade ao assinarem o "Plano de Açáo de cinco anos para a prevençáo da obesidade em crianças e adolescentes". Dentre outras medidas, o plano pre- vê a melhoria da nutrição escolar e atividade física e a promoção da alimentação saudável e uma das soluçóes propostas são ações de educação em saúde voltada para o público infantil. ${ }^{(4,6,7)}$

A educação em saúde é uma forma de melhorar a compreensáo do usuário à doença, o que, por sua vez, pode melhorar seu estado geral e reduzir a utilização dos recursos de saúde. ${ }^{(8)}$ A partir disso, têm-se criado intervençóes educativas, com atributos inovadores e mais adequados ao público-alvo. Uma dessas abordagens tem como base o uso de serious games (jogos sérios) como um meio de melhoria de conhecimentos e como uma ferramenta adicional em programas de educação e promoção da saúde. ${ }^{(9)}$

De acordo com um dos precursores dessa terminologia, um serious game é um jogo no qual há um objetivo de aprendizado a ser alcançado. Entretanto, isso não significa que esse tipo de jogo seja desinteressante ou menos divertido, apenas reforça que existe um propósito além da diversão. ${ }^{(10)}$

Os programas de combate e tratamento da obesidade infantil enfocam em sua maioria na melhoria de comportamentos alimentares e aumento da atividade física, ${ }^{(11)}$ contudo abordar o público infantil em seu ambiente familiar com novas estratégias e mídias, como os serious games, pode trazer um diferencial importante para melhor adesão desses programas.

Os jogos podem oferecer a chance de ensinar as crianças a enfrentar o sobrepeso e a obesidade, já que esses têm caráter altamente convidativo a esse público, o que facilita o alcance e interesse das crianças. ${ }^{(12)}$ Seus elementos aumentam o interesse, a motivação e o prazer, melhorando assim o engajamento das mesmas dentro dos programas. ${ }^{(13)}$

Nesse escopo, este artigo apresenta o jogo DigesTower, um serious game voltado para o público 
infantil, com vistas a incentivar a alimentação saudável e o exercício físico. O objetivo principal deste estudo foi avaliar o serious game enquanto estratégia educativa para crianças em um programa de enfrentamento de sobrepeso e obesidade.

\section{Métodos}

Tratou-se de uma pesquisa de abordagem quantitativa, metodológica e transversal. A pesquisa metodológica realiza o desenvolvimento, validação ou avaliação de ferramentas e métodos de pesquisa. ${ }^{(14)}$

O serious game DigesTower foi desenvolvido e avaliado por especialistas de saúde e de computação em uma etapa anterior, no ano de 2014. Posteriormente, o jogo passou por uma reformulação para adequá-lo e apresentá-lo futuramente ao público-alvo. ${ }^{(15)}$ Seu desenvolvimento contou com a colaboração de uma equipe multiprofissional e foi embasado nos estudos de Novak ${ }^{(16)}$ e Schell, ${ }^{(17)}$ bem como nas teorias de mudança de comportamento em saúde. ${ }^{(18)}$

Os temas principais do jogo são a alimentação saudável e o exercício físico e busca incentivar melhores hábitos alimentares. O sistema digestório humano, seus principais órgãos e enzimas digestivas são apresentados de forma lúdica e o serious game explora o processo de digestáo de cada classe alimentar (carboidratos, proteínas e gorduras).

O Diges Tower é classificado como Tower Defense, ou seja, um subtipo dos jogos de estratégia que, como o nome já sugere, é focado na defesa de um elemento do jogo. ${ }^{(19)}$ No caso deste jogo em específico, os alimentos virão em ondas e precisam ser digeridos pelas enzimas digestivas.

A partir disso, o serious game DigesTower foi apresentado para crianças escolares a fim de ser avaliado enquanto estratégia educativa. Os participantes já eram acompanhados, desde janeiro de 2017, em um programa de condicionamento físico com jogos interativos para crianças obesas e/ou sobrepeso desenvolvido em uma Universidade Estadual do interior do Estado de Sáo Paulo. O programa conta com três frentes: acompanhamento médico e nutricional, acompanhamento psicológico e atividades físicas. As crianças participantes do programa não possuíam outras comorbidades associadas ao sobrepeso e obesidade e praticavam atividade física exclusivamente no período escolar.

O estudo foi encaminhado e aprovado pelo Comitê de Ética em Pesquisa (CEP) da Universidade. Os pais e crianças foram convidados a participar da pesquisa através de contato presencial e foi solicitada a assinatura dos Termos de Consentimento Livre e Esclarecido (TCLE) e do Termo de Assentimento. Em seguida, as crianças receberam o serious game como uma estratégia adicional, além das estratégias usuais já utilizadas pelo programa.

A primeira etapa da pesquisa consistiu na avaliação antropométrica das crianças e foram mensuradas suas medidas, peso, altura e Índice de Massa Corpórea (IMC) e classificação por Escore Z, de acordo com procedimentos e gráficos disponibilizados e recomendados pela Organização Mundial de Saúde (OMS). ${ }^{(20)}$ Seguiu-se com a aplicação de instrumentos para coleta de dados com o Questionário de Alimentação do Dia Anterior - QUADA ${ }^{(21)}$ e o Questionário de Atividade Física do Dia Anterior - QUAFDA ${ }^{(22)}$ para avaliar o consumo alimentar e a prática de exercícios físicos, o Child Eating Behaviour Questionnaire $\mathrm{CEBQ}^{(23)}$ para verificar o comportamento alimentar dos escolares e por fim o EGameFlow ${ }^{(24)}$ para avaliação do serious game proposto.

Realizou-se um levantamento do perfil das crianças pertencentes ao programa. Após a mensuração dos dados antropométricos foram aplicados questionários diretamente às crianças a respeito dos seus hábitos alimentares e de atividade física nas últimas 24 horas, considerando sempre a aplicação em dias de semana em que há uma alimentação e atividades físicas comuns na rotina da família, ou seja, evitando as coletas em uma semana com feriados ou dias posteriores ao final de semana como a segunda-feira.

No instrumento QUADA há uma disposição de refeições, ordenadas cronologicamente: café, lanche da manhã, almoço, lanche da tarde, jantar e lanche da noite. Cada refeiçáo é ilustrada com 21 alimentos diversos. ${ }^{(21)}$ Tais alimentos foram selecionados considerando-se os padróes alimentares das crianças da faixa etária escolar, a disponibilidade de alimentos, 
o cardápio oferecido em escolas e o Guia Alimentar para a População Brasileira. ${ }^{(25)}$

$\mathrm{O}$ instrumento QUAFDA verifica a atitude da criança em relação à atividade física, o meio de transporte utilizado para ir à escola, e as atividades físicas praticadas. $\mathrm{O}$ escore geral de atividade física da criança nesse questionário é determinado ao somar os escores das atividades que a criança referiu realizar no dia anterior, baseado nos desenhos de 11 atividades pré-definidas e com três intensidades diferentes. $\mathrm{O}$ nível de atividade física foi avaliado pelos tercis de distribuição do escore gerado pelo instrumento. Os valores de distribuição do escore QUAFDA em tercis foram 35, 58 e 141, respectivamente, para o $1^{\circ}, 2^{\circ}$ e $3^{\circ}$ tercis. Para caracterizar o padrão de atividade física apresentadas no questionário, os participantes foram classificados em menos ativos, intermediários e mais ativos. ${ }^{(22)}$

Apenas o questionário CEBQ foi destinado aos familiares para avaliação do comportamento alimentar por instrução dos próprios autores do instrumento. O CEBQ contém 35 itens e objetiva avaliar oito dimensóes do comportamento alimentar: resposta à saciedade, ingestão lenta, seletividade, resposta à comida, prazer em comer, desejo de beber, sobre-ingestão emocional e sub-ingestão emocional. Cada item é avaliado numa escala de tipo Likert de cinco pontos que variam entre 1-nada frequente e 5-muito frequente. Soma-se a pontuação das perguntas pertencentes à mesma dimensão, de forma que cada uma delas apresentará um valor médio e desvio-padrão. ${ }^{(23)}$

Posteriormente, o serious game DigesTower foi apresentado ao final das atividades do programa de condicionamento físico, de maneira individual. Foram realizadas duas sessóes, em dias distintos, ambas com 30 minutos. Ao final, a criança respondia o questionário EgameFlow para a avaliação do jogo. ${ }^{(24)}$

Esse instrumento procura criar uma escala de satisfação do usuário com o jogo educacional. $\mathrm{O}$ avaliador atribui uma nota de um a sete a cada item presente nas oito categorias (Concentração, Desafios, Autonomia, Clareza dos objetivos, Feedback, Imersão e Melhoria do Conhecimento), sendo o valor um considerado "fraco" e o sete considerado o mais "forte". (24)
Os dados obtidos na pesquisa foram organizados no software Excel e analisados quantitativamente por meio por estatística descritiva, tais como frequência simples, média, mediana, desvio-padrão. ${ }^{(26)}$ Os dados foram organizados em tabela, gráficos e porcentagens de acordo com seus itens e categorias, bem como avaliados e analisados à luz da teoria sobre a temática do estudo. ${ }^{(21-25)}$

\section{Resultados}

Todas as crianças do programa de condicionamento físico com jogos interativos para crianças obesas e/ou com sobrepeso foram convocadas e aceitaram participar do estudo, totalizando 13 crianças. A coleta de dados ocorreu de janeiro a julho de 2017. Os dados antropométricos das crianças participantes estão apresentados na tabela 1 .

Tabela 1. Caracterização das crianças participantes no estudo $\left(n^{*}=13\right)$

\begin{tabular}{|c|c|c|c|c|c|c|}
\hline Criança & Sexo & $\begin{array}{l}\text { Idade } \\
\text { (anos) }\end{array}$ & $\begin{array}{c}\text { Massa } \\
\text { corporal } \\
\text { (Kilograma) }\end{array}$ & $\begin{array}{l}\text { Estatura } \\
\text { (metros) }\end{array}$ & $\begin{array}{c}\text { IMC† } \\
\text { (Kilograma } \\
\text { /metros }{ }^{2} \text { ) }\end{array}$ & Escore Z \\
\hline C1 & $F$ & 9 anos & 74,5 & 1,54 & 30,6 & $\begin{array}{c}>+3 \text { (obesidade } \\
\text { grave) }\end{array}$ \\
\hline C2 & $M$ & 8 anos & 47,9 & 1,39 & 24,8 & $\begin{array}{c}>+3 \text { (obesidade } \\
\text { grave) }\end{array}$ \\
\hline C3 & M & 8 anos & 63,6 & 1,37 & 33,9 & $\begin{array}{c}>+3 \text { (obesidade } \\
\text { grave) }\end{array}$ \\
\hline C4 & $M$ & 9 anos & 52,1 & 1,39 & 26,9 & $\begin{array}{c}>+3 \text { (obesidade } \\
\text { grave) }\end{array}$ \\
\hline C5 & $\mathrm{M}$ & 10 anos & 92,9 & 1,55 & 38,6 & $\begin{array}{c}>+3 \text { (obesidade } \\
\text { grave) }\end{array}$ \\
\hline C6 & $M$ & 10 anos & 70,2 & 1,56 & 29,2 & $\begin{array}{c}>+3 \text { (obesidade } \\
\text { grave) }\end{array}$ \\
\hline $\mathrm{C7}$ & $\mathrm{M}$ & 11 anos & 111,5 & 1,66 & 40,4 & $\begin{array}{c}>+3 \text { (obesidade } \\
\text { grave) }\end{array}$ \\
\hline C8 & $\mathrm{F}$ & 8 anos & 53,8 & 1,43 & 26,3 & $\begin{array}{c}>+3 \text { (obesidade } \\
\text { grave) }\end{array}$ \\
\hline C9 & $M$ & 11 anos & 58,1 & 1,44 & 28 & $\begin{array}{c}>+2<+3 \\
\text { (obesidade) }\end{array}$ \\
\hline $\mathrm{C} 10$ & M & 8 anos & 40,6 & 1,39 & 21,1 & $\begin{array}{c}>+2<+3 \\
\text { (obesidade) }\end{array}$ \\
\hline $\mathrm{C} 11$ & $M$ & 8 anos & 45,2 & 1,4 & 23 & $\begin{array}{c}>+2<+3 \\
\text { (obesidade) }\end{array}$ \\
\hline C12 & M & 7 anos & 41 & 1,34 & 22,8 & $\begin{array}{c}>+2<+3 \\
\text { (obesidade) }\end{array}$ \\
\hline $\mathrm{C} 13$ & $\mathrm{M}$ & 7 anos & 39,9 & 1,32 & 22,9 & $\begin{array}{c}>+2<+3 \\
\text { (obesidade) }\end{array}$ \\
\hline
\end{tabular}

*n - número de participantes (frequência absoluta); †IMC - Índice de Massa Corpórea; † Escore Z Escore padrão

Foi aplicado o questionário QUADA para avaliação do consumo alimentar nas últimas 24 horas. As proporçóes das crianças que atenderam às recomendaçóes estão apresentadas na figura 1 . 


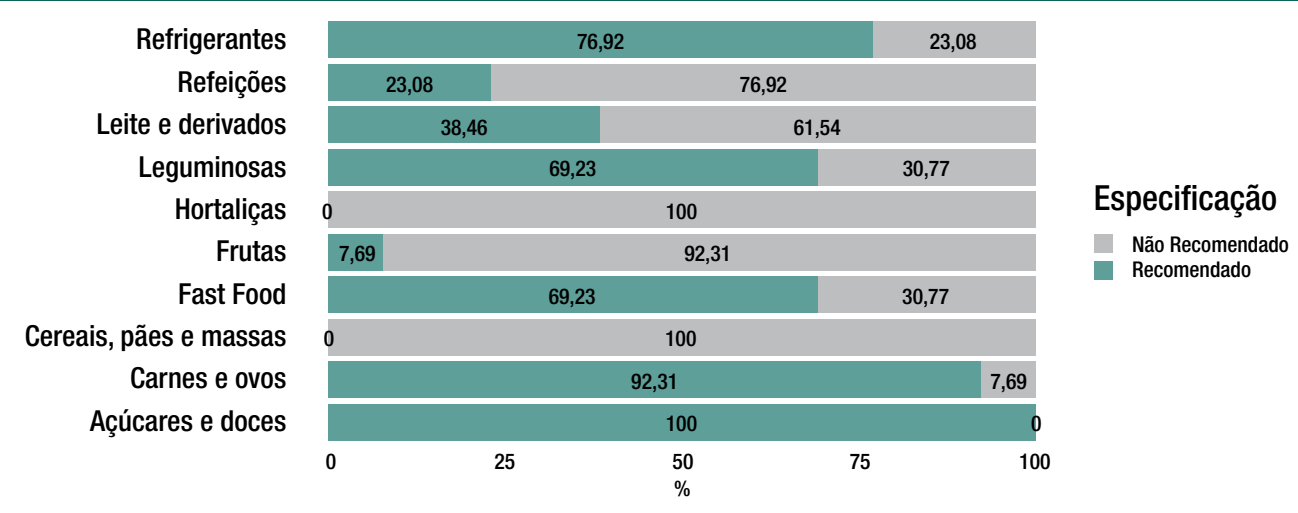

Figura 1. Porcentagem de crianças que ingerem as porções diárias recomendadas pelo Guia Alimentar para a População Brasileira $(n=13)$

Há o consumo de cereais, pães e massas pelas crianças, porém o consumo de todas as crianças ficou muito abaixo do recomendado. O mesmo aconteceu com o consumo de hortaliças, nenhum dos participantes atingiu o recomendado pelo guia. Entretanto, um dado positivo foi que apesar de não ingerirem muitas hortaliças, a maioria das crianças consome leguminosas $(69,23 \%)$, especialmente o feijão em refeiçóes principais, como almoço e jantar.

Do total de 13 crianças, 10 participantes relataram náo consumir lanches com alto teor de gordura (batatas fritas, pizza e hambúrguer), bem como refrigerantes no dia anterior à administração do questionário. As crianças também não abusaram do recomendado de açúcares e doces, ingerindo até uma porção desse grupo alimentar. No entanto, o consumo de frutas foi muito abaixo do desejado, a maioria não consumiu nenhuma porção de fruta e somente uma criança atingiu os valores recomendados de três porçóes ao dia $(7,1 \%)$.

Após, foi aplicado o questionário QUAFDA para avaliação das atividades físicas praticadas nas últimas 24 horas. Do total de participantes, 10 crianças foram classificadas como menos ativas $(76,92 \%)$, três como intermediárias $(23,08 \%)$ e não houve nenhuma criança que chegou ao escore de nível alto de atividade física de acordo com o questionário, considerada mais ativa.

No que se referem aos comportamentos alimentares das crianças, esses foram analisados através do Child Eating Behaviour Questionnaire (CEBQ). A figura 2 ilustra as médias das respostas das crianças participantes no questionário CEBQ.

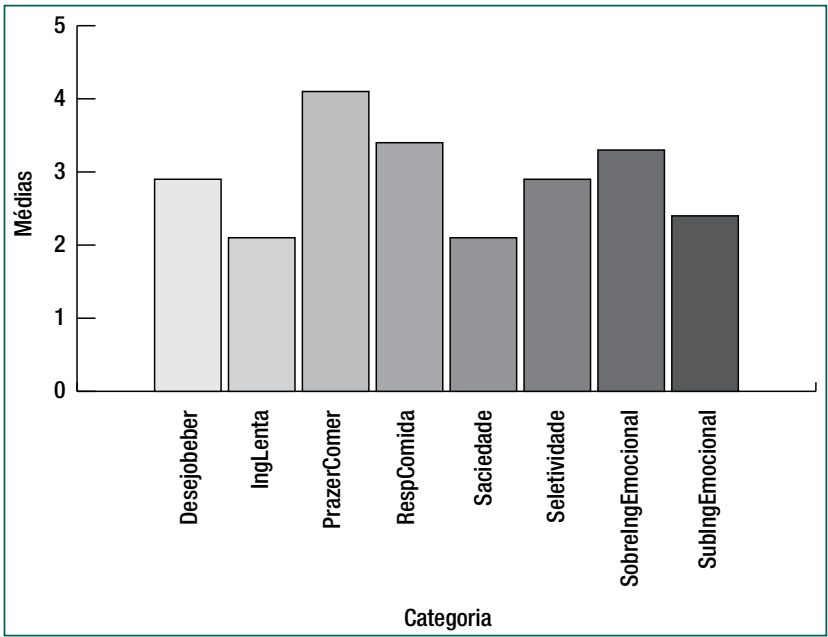

Figura 2. Médias das respostas em cada dimensão do CEBQ ( $n=13)$

As médias mais altas estão presentes na dimensão "Resposta à comida" e "Prazer em comer", enquanto as mais baixas se referem às dimensôes "Resposta à saciedade" e "Ingestão lenta”. Outra média baixa foi vista na dimensão "Sub ingestão emocional". Após responderem todos os instrumentos para a realização do perfil das crianças, o serious game DigesTower foi apresentado às mesmas. Após finalizarem o jogo as crianças foram convidadas a responder um questionário para avaliação do mesmo. Utilizou-se uma versáo adaptada do questionário EGameFlow, questionário específico para avaliaçôes de jogos educacionais. As avaliaçôes feitas pelas crianças foram agrupadas por categoria e estão descritas na tabela 2 .

$\mathrm{O}$ jogo foi bem recebido pelas crianças, que afirmaram ter tido imersão, motivação e estímulo para melhoria do seu conhecimento. Isso pôde ser percebido através das altas médias das respostas nas diversas categorias. Foi possível notar que as cate- 
Tabela 2. Estatísticas descritivas relativas às categorias do EGameFlow $\left(n^{\star}=13\right)$

\begin{tabular}{l|c|c|c|c}
\hline $\begin{array}{l}\text { Categorias do } \\
\text { EGameFlow }\end{array}$ & $\begin{array}{c}\text { Mínimo- } \\
\text { Máximo }\end{array}$ & Mediana & Média & Desvio-padrão \\
\hline Concentração & $4,00-7,00$ & 7,00 & 6,38 & 0,89 \\
Desafios & $5,75-7,00$ & 6,50 & 6,54 & 0,50 \\
Autonomia & $3,75-7,00$ & 6,75 & 6,08 & 1,20 \\
Clareza & $4,00-7,00$ & 7,00 & 6,46 & 1,01 \\
Feedback & $4,00-7,00$ & 7,00 & 6,38 & 1,02 \\
Imersão & $5,50-7,00$ & 7,00 & 6,71 & 0,49 \\
Melhoria do conhecimento & $4,33-7,00$ & 6,67 & 6,13 & 1,03 \\
\hline
\end{tabular}

$\mathrm{n}^{\star}$ - número de participantes (frequência absoluta)

gorias "Desafios" e "Imersão" além de possuírem as maiores médias do questionário, foram as que possuíram os menores desvios-padrão, ou seja, a maioria das crianças pontuou de forma semelhante esses temas, com notas seis e sete. Destaca-se na categoria "Desafios" a maior nota dada pelas crianças, referente às habilidades do jogo e o quanto elas aumentam ao longo do mesmo. Contudo, foi preciso rever a questão quatro. A pergunta abordava a dificuldade do jogo e houve uma variação das respostas, algumas crianças acharam a dificuldade adequada e outros consideraram o jogo difícil. Entretanto, todas as crianças conseguiram superar as dificuldades impostas no DigesTower e consequentemente finalizá-lo. A categoria "Imersão" foi a categoria melhor avaliada pelas crianças. Notou-se que as crianças ficaram bastante imersas no jogo e demonstraram um grande envolvimento com a atividade. Já as categorias "Autonomia" e "Melhoria do Conhecimento" obtiveram as médias mais baixas quando comparadas às demais e possuíram os desvios-padrão maiores, indicando que provavelmente algumas crianças deram pontuaçóes baixas enquanto outras deram pontuação alta. Com relação aos itens avaliados na "Autonomia", as crianças referiram não ter muito controle do menu (questáo sete), evidenciado pela proporção de notas abaixo de seis (23\%). Apesar disso, houve uma avaliação positiva quanto à recuperação de erros cometidos ao longo do jogo.

Outra dificuldade levantada pelas crianças foi vista nas respostas da questão 10 ("Sei o próximo passo no jogo?”), pertencente à categoria Autonomia, que obteve quatro notas abaixo de seis e foi preciso ser revisto para a versão final do DigesTower. Fez-se necessário uma explicação mais clara na interface sobre o que viria a acontecer nas próximas etapas do jogo.
A maioria das crianças avaliou positivamente a categoria "Feedback", porém nessa categoria a Questão 13 ("Recebo feedback do meu progresso no jogo?") obteve notas mais baixas (23\%) e também foi preciso repensar a dinâmica de feedbacks do mesmo, a fim de melhorá-lo. Um exemplo utilizado para esse fim foi a adição de mais efeitos audiovisuais para alertar ao jogador algumas açóes durante o jogo. A análise dos dados oriundos do EgameFlow contribuiu para que as necessidades de adequaçóes fossem apontadas e contempladas na versão final do serious game.

\section{Discussão}

Neste estudo houve a inserção do serious game DigesTower em um programa interdisciplinar de enfrentamento da obesidade infantil. Os IMCs das crianças participantes apresentaram valores de escores $\mathrm{Z}$ referentes à obesidade e obesidade grave. Contudo, esses valores eram esperados, uma vez que era um pré-requisito a criança estar acima da eutrofia para a sua participação no programa de condicionamento físico com jogos interativos para crianças obesas e/ou sobrepeso. Ademais, a literatura também reforça que há uma tendência significativa de aumento da prevalência da obesidade em crianças e adolescentes atualmente. ${ }^{(27)}$

Para a discussão sobre a alimentação do dia anterior, os resultados deste estudo ilustram que houve uma baixa porcentagem de crianças que consumiu salgadinhos e refrigerantes no dia anterior (cerca de $30 \%$ ) e essa informação está em discordância com os dados relatados na literatura para crianças e adolescentes do Brasil e de outros países, uma vez que são porcentagens maiores. ${ }^{(28,29)}$

Acredita-se que os dados encontrados neste estudo sejam menores devido à inserção das crianças no programa de condicionamento físico da Universidade, que provê consultas com médicos pediatras e nutricionistas que visam à mudança dos hábitos alimentares e todas as crianças do grupo já se encontravam em acompanhamento e mantinham uma dieta específica para doenças metabólicas.

Já se tratando dos dados encontrados sobre a atividade física das crianças no dia anterior, os resulta- 
dos também discordam do exposto na literatura, pois há uma distribuição mais equilibrada em outro estudo que utilizou o mesmo instrumento, apresentando como dados $23,8 \%$ de crianças menos ativas, $36,7 \%$ de intermediárias e 39,5\% mais ativas, diferentemente do encontrado que são somente crianças menos ativas $(76,92 \%)$ e intermediárias $(23,08 \%)$. $^{(22)}$

Além disso, foi utilizado neste estudo o questionário CEBQ para obtenção de dados referentes aos hábitos alimentares dos participantes e notaram-se médias maiores na dimensão "Resposta à comida" (FR) e "Prazer em comer" (EF), enquanto as médias mais baixas se referem às dimensóes "Resposta à saciedade" (SR) e "Ingestão lenta" (SE), o que vai de acordo com a literatura. Alguns autores comentam que as crianças com excesso de peso exibiram menor pontuação na subescala "Ingestão lenta", o que demonstra um padrão de alimentação mais rápido. ${ }^{(30)}$

Ainda, os resultados dessas subescalas encontram-se, geralmente, elevados nas crianças obesas e tendem a ser baixos em crianças com baixo peso. ${ }^{(23)}$ Os valores mais altos nas dimensôes "Resposta à comida" e "Prazer em comer" estão de acordo com o esperado pela literatura, visto que foram avaliadas somente com crianças com sobrepeso ou obesidade.

Outra média baixa foi vista na dimensão "Resposta à saciedade". De acordo com os autores da escala, essa dimensão avalia a capacidade de regulação do apetite de modo a compensar uma refeição anterior. Uma resposta baixa à saciedade é classificada entre as prováveis causas comportamentais da obesidade. ${ }^{(23)}$

Nesse âmbito, devido ao rápido avanço da tecnologia nas últimas décadas, a literatura aponta que a geração atual tem necessidades de aprendizagem diferenciadas, quando comparados às geraçóes passadas. Estes aprendem muito mais facilmente quando o aprendizado se relaciona com a tecnologia. ${ }^{(31)}$

A respeito da avaliação de serious games, alguns autores afirmam ser essencial a obtenção de Feedback do público-alvo. Seus comentários são muito pertinentes para refinar os componentes do jogo e aprimorar sua adequação. ${ }^{(32)}$

Alguns estudos têm observado que os serious games trazem efeitos positivos para o desenvolvimento de estilos de vida saudáveis, porém o que mais se destaca nos estudos é a melhoria no conhecimento. Efeitos sobre os resultados clínicos foram significativos, mas muito menores. Em geral, esses jogos aumentaram a adoção de estilo de vida saudável, embora os tamanhos dos efeitos sejam pequenos. ${ }^{(33)}$

Outro dado importante destacado pela literatura é que os jogadores estão mais dispostos a gastar tempo de aprendizado em jogos do que aprendendo através de métodos tradicionais, desde que o desafio no jogo seja viável. Assim sendo, os serious games podem ser um meio melhor do que outras intervençôes realizadas por computador para atingir o público-alvo em uma implementação em larga escala. ${ }^{(33)}$

A respeito da avaliação do jogo DigesTower, as crianças referiram não ter muito controle do menu evidenciado pela proporçáa de notas abaixo de seis (23\%). Tais notas podem ter ocorrido por se tratar de um jogo de tower defense e esse tipo de jogo possui momentos de apenas observação das açóes realizadas, o que pode ter gerado essa sensação de pouco controle do jogo em si. Apesar disso, elas avaliaram positivamente a recuperação de erros cometidos durante o jogo e sentiram que podiam utilizar de várias estratégias.

Outra dificuldade encontrada pelas crianças foi na categoria Autonomia evidenciada nas respostas da questão 10 (“Sei o próximo passo no jogo?”), que obteve $31 \%$ de notas abaixo de seis e foi necessária uma revisão para a versão final do jogo. Foi preciso deixar mais claro na interface o que iria acontecer na próxima etapa, como, por exemplo, adicionar na tela o quadro "próximo alimento", que ilustrava o alimento que iria aparecer logo em seguida, visando alertar ao jogador para que ele se preparasse melhor para enfrentá-lo.

Finalmente, como limitação deste estudo há o fato de a coleta dos dados ter sido feita com uma quantidade limitada de crianças, pois se considerou somente a população que estava participando do "programa de condicionamento físico com games interativos para crianças obesas e/ou sobrepeso" e que eram crianças que já se encontravam com sobrepeso ou obesidade.

Obter avaliaçôes de crianças que não se integram a esse grupo pode evidenciar necessidades ou sugestóes diferentes daquelas identificadas nesta pesquisa e enriquecer ainda mais a avaliação do serious game em questáo. 


\section{Conclusão}

Ao final do estudo foi possível alcançar os objetivos propostos, delinear o perfil das crianças participantes em um programa de enfrentamento da obesidade, bem como avaliar junto a esse público o serious game DigesTower enquanto estratégia educativa. O DigesTower foi considerado adequado se destacou como uma ferramenta tecnológica inovadora. Busca-se futuramente utilizar o serious game como uma possível ferramenta de intervenção à obesidade infantil. Identificou-se, por meio das avaliaçóes do público-alvo, que o DigesTower apresentou potencial enquanto ferramenta educacional, buscando incentivar a criança a melhores hábitos e motivar sua adesão ao programa de tratamento da obesidade. Também, este estudo foi desenvolvido a fim de contribuir com a área de tecnologias para a saúde, mais especificamente para o desenvolvimento de novas estratégias para a educação e promoção da saúde, demonstrando que esse tipo de ferramenta possa não só ser prazerosa como também eficaz. Por fim, a pesquisa corrobora com a literatura da área ao lançar algo do interesse dessa geração de crianças, trazendo uma inovação tecnológica e buscando utilizar tecnologias e dispositivos que já são parte do dia-a-dia desse público.

\section{Agradecimentos}

Agradecemos às agências de fomento: Conselho Nacional de Ciência e Tecnologia (CNPq; bolsa de doutorado para a autora Jéssica David Dias); à Coordenação de Aperfeiçoamento de Pessoal de Nível Superior (CAPES; bolsa de mestrado para a autora Rafaella Belém Aragão e bolsa de doutorado para a autora Chris Mayara Tibes-Cherman).

\section{Colaborações}

Dias JD, Tibes-Cherman CM, Aragão RB, Tourinho Filho H, Zem-Mascarenhas SH, Fonseca LMM contribuíram com a concepção do estudo, análise e interpretação dos dados, redação do artigo, revisão crítica relevante do conteúdo intelectual e aprovação da versão final a ser publicada.

\section{Referências}

1. Associação Brasileira para o Estudo da Obesidade e da Síndrome Metabólica (ABESO). Diretrizes Brasileiras de Obesidade. 4a ed. São Paulo: ABESO; 2016. [citado 2020 Set 26]. Disponível em: https:// abeso.org.br/wp-content/uploads/2019/12/Diretrizes-DownloadDiretrizes-Brasileiras-de-Obesidade-2016.pdf

2. NCD Risk Factor Collaboration (NCD-RisC). Worldwide trends in body-mass index, underweight, overweight, and obesity from 1975 to 2016: a pooled analysis of 2416 population-based measurement studies in 128.9 million children, adolescents, and adults. Lancet. 2017;390(10113):2627-2642.

3. Lobstein T, Jackson-Leach R. Planning for the worst: estimates of obesity and comorbidities in school-age children in 2025. Pediatr Obes. 2016;11(5):321-5.

4. Pan American Health Organization (PAHO). World Health Organization (WHO). Plan of action for the prevention of obesity in children and adolescents. Washington (DC): PAHO; WHO: 2014 Oct 03 [cited 2020 Apr 24]. Available from: https://www.paho.org/hq/dmdocuments/2014/CD53-9-e.pdf

5. Rivera JÁ, de Cossío TG, Pedraza LS, Aburto TC, Sánchez TG, Martorell R. Childhood and adolescent overweight and obesity in Latin America: a systematic review. Lancet Diabetes Endocrinol. 2014;2(4):321-32. Review.

6. Brazil. Ministério da Saúde. Ministério da Educação. Caderno do gestor do PSE. Brasília (DF): Ministério da Saúde; 2015 [citado 2020 Jan 20]. Disponível em: http://bvsms.saude.gov.br/bvs/publicacoes/caderno_ gestor_pse.pdf

7. World Health Organization (WHO). Report of the commission on ending childhood obesity. Geneva: WHO; 2016 [cited 2020 Jan 12]. Available from: https://apps.who.int/iris/bitstream/ handle/10665/204176/9789241510066_eng.pdf

8. Charlier N, Zupancic N, Fieuws S, Denhaerynck K, Zaman B, Moons P. Serious games for improving knowledge and self-management in young people with chronic conditions: a systematic review and metaanalysis. J Am Med Inform Assoc. 2016;23(1):230-9. Erratum in: J Am Med Inform Assoc. 2018;25(9):1270-1. Review.

9. Rocha RV, Zem-Lopes AM, Pedro LZ, Bittencourt II, Isotani S. Metodologia de Desenvolvimento de Jogos Sérios: especificação de ferramentas de apoio open source. Braz J Computers Education. 2016;24(3):109.

10. Dörner R, Göbel S, Effelsberg W, Wiemeyer J, editors. Serious Games. 4th ed. New York: Viking Press; 1987.

11. Mack I, Sauer H, Weimer K, Dammann D, Zipfel S, Enck P, et al. Obese children and adolescents need increased gastric volumes in order to perceive satiety. Obesity (Silver Spring). 2014;22(10):2123-5.

12. Gao Z, Chen S, Pasco D, Pope Z. A meta-analysis of active video games on health outcomes among children and adolescents. Obes Rev. 2015;16(9):783-94.

13. Brown M, O'Neill N, van Woerden $H$, Eslambolchilar $P$, Jones $M$, John $A$. Gamification and adherence to web-based mental health interventions: a systematic review. JMIR Ment Health. 2016;3(3):e39.

14. Polit $D$, Beck $C$. Fundamentos da pesquisa em enfermagem: avaliação de evidências para a prática de enfermagem. 9th ed. Porto Alegre: Artmed; 2019.

15. Dias JD, Mekaro MS, Cheng Lu JK, Otsuka JL, Fonseca LM, ZemMascarenhas SH. Serious game development as a strategy for health promotion and tackling childhood obesity. Rev Lat Am Enfermagem. 2016;24:e2759. 
Dias JD, Tibes-Cherman CM, Aragão RB, Tourinho Filho H, Zem-Mascarenhas SH, Fonseca LM

16. Novak J. Game development essentials. 2nd ed. USA: Delmar Cengage Learning; 2010.

17. Schell J. The art of game design: a book of lenses. Massachusetts: AK Peters; 2014

18. Prochaska J0, Velicer WF. The transtheoretical model of health behavior change. Am J Health Promot. 1997;12(1):38-48.

19. Zechner M, DiMarzio JF, Green R. Beginning Android Games. Berkeley (CA): Apress; 2016.

20. World Health Organization (WHO). WHO Child growth standards: length/ height for age, weight-for-age, weight-for-length, weight-for-height and body mass index-for-age, methods and development. Geneva: WHO; 2006 [cited 2019 Nov 20]. Available from: https://apps.who.int/ iris/bitstream/handle/10665/43413/924154693X_eng.pdf

21. Assis MA,BenedetJ, Kerpel R, Vasconcelos FA, DiPietroPF,KupekE. Validação da terceira versão do Questionário Alimentar do Dia Anterior (QUADA-3) para escolares de 6 a 11 anos. Cad Saúde Pública. 2009;25:1816-26.

22. Costa FF, Assis MA. Nível de atividade física e comportamentos sedentários de escolares de sete a dez anos de Florianópolis-SC. Rev Bras Ativ Fís Saúde. 2011;16(1):48-54.

23. Viana V, Sinde S, Saxton JC. Children's Eating Behaviour Questionnaire: associations with BMI in Portuguese children. Br J Nutr. 2008;100(2):445-50.

24. Fu FL, Su RC, Yu SC. EGameFlow: a scale to measure learners' enjoyment of e-learning games. Comput Educ. 2009;52(1):101-12.

25. Brazil. Ministry of Health of Brazil. Secretariat of Health Care. Primary Health Care Department. Dietary Guidelines for the Brazilian population. Brasilia (DF): Ministry of Health; 2015 [cited 2020 May 3]. Available from: http://bvsms.saude.gov.br/bvs/publicacoes/dietary_guidelines_ brazilian_population.pdf

26. Pedroso B, Pilatti LA, Gutierrez GL, Picinin CT. Cálculo dos escores e estatística descritiva do WHOQOL-bref através do Microsoft Excel. Rev Bras Qualidad Vida. 2010;2:31-3.

27. Flores LS, Gaya AR, Petersen RD, Gaya A. Trends of underweight, overweight, and obesity in Brazilian children and adolescents. J Pediatria (Rio J). 2013;89(5):456-61.

28. Assis MA, Calvo MC, Kupek E, Vasconcelos FD, Campos VC, Machado $\mathrm{M}$, et al. Qualitative analysis of the diet of a probabilistic sample of schoolchildren from Florianópolis, Santa Catarina State, Brazil, using the Previous Day Food Questionnaire. Cad Saúde Pública. 2010;26(7):1355-65.

29. Sichieri R, Paula Trotte A, de Souza RA, Veiga GV. School randomised trial on prevention of excessive weight gain by discouraging students from drinking sodas. Public Health Nutr. 2009;12(2):197-202.

30. Passos DR, Gigante DP, Maciel FV, Matijasevich A. Children's eating behavior: comparison between normal and overweight children from a school in Pelotas, Rio Grande do Sul, Brazil. Rev Paul Pediatr. 2015;33(1):42-9.

31. Pick AM, Begley KJ, Augustine S. Changes in teaching strategies to accommodate a new generation of learner: a case study. Pharmacy Educ. 2017;17(1):95-99.

32. Thompson D. What serious video games can offer child obesity prevention. JMIR Serious Games. 2014;2(2):e8.

33. DeSmet A, Van Ryckeghem D, Compernolle S, Baranowski T, Thompson D, Crombez $G$, et al. A meta-analysis of serious digital games for healthy lifestyle promotion. Prev Med. 2014;69:95-107. 